\title{
Geographic Variation in Body Size and Weight of Willow Ptarmigan
}

\author{
GEORGE C. WEST ${ }^{1}$, ROBERT B. WEEDEN ${ }^{2}$, \\ LAURENCE IRVING ${ }^{1}$ and LEONARD J. PEYTON ${ }^{1}$
}

\begin{abstract}
Multiple range test comparisons of wing, tail, and net body weight measurements of 2,600 willow ptarmigan (Lagopus lagopus) specimens collected in Alaska and adjacent Yukon Territory showed statistically significant differences among populations from different geographic areas but also indicated surprising uniformity within populations designated as recognizable subspecies.

Previous range distributions based on summer plumage colour and size of bill separated L. l. alascensis from L. l. albus at the Alaska-Yukon border. On the basis of our measurements, it is believed that the population of the larger $L$. l.alascensis extends eastward into Yukon Territory at least to Old Crow whereas the smaller L. $l$. albus extends westward into Alaska in the upper Tanana River Valley and south of the Alaska Range to the Susitna River. It is suggested that the present distribution of the various subspecies of willow ptarmigan in Alaska may be explained in part by their distribution at the time of Wisconsin glaciation and their subsequent dispersal.
\end{abstract}

RÉSUMÉ. Variations géographiques de la taille et du poids du Lagopède des saules. De multiples comparaisons de mesures des ailes, de la queue et du poids net de 2,600 spécimens de lagopède des saules (Lagopus lagopus) recueillis en Alaska et dans la partie adjacente du territoire du Yukon, ont démontré des différences statistiquement significatives entre les populations des différentes aires géographiques, mais ont aussi indiqué une surprenante uniformité parmi les populations désignées comme sous-espèces reconnaissables.

Les précédentes distributions d'habitat, basées sur la couleur du plumage et la taille du bec, distinguaient $L$. $l$. alascensis de $L$. $l$. albus à la frontière AlaskaYukon. Sur la base des nouvelles mesures, on croit que la population du L. $l$. alascensis plus gros s'étend vers l'Est dans le territoire du Yukon au moins jusqu'à Old Crow, tandis que le $L$. $l$. albus plus petit s'étend vers l'Ouest à travers l'Alaska, dans la haute vallée de la Tanana et au Sud de la chaîne alaskienne jusqu’à la Susitna. On suppose que la distribution présente des diverses sous-espèces du Lagopède des saules en Alaska peut s'expliquer par leur distribution à l'époque de la glaciation wisconsinienne et par leur dispersion subséquente.

РЕ3ЮМЕ. Различия в размере и весе тела куропатки белой. Данные многочисленных определений длины крыльев і хвоста, а также веса тела 2600 особей куропатки белой (Lagopus lagopus), проведенных в Аляске и на прилегающей к ней территории Юкона, выявили статистически значительные различия в әтих параметрах среди популяций, обитающих в различных географических областях. В то же время была отмечена значительная однородность перечисленных характеристик среди популяций, выделенных как определенные подвиды.

На основе окраски летнего оперения и величины клюва куропатки белой, предыдущие исследователи разделяли ареалы L. 1. alascensis и L. 1. albus вдоль границы между Аляской и Юконом. Проведенныв исследования позволили уточнить границы этих ареалов. Предполагается, что современное распределение различных подвидов куропатки белой можно частично объяснить на основе их распределения в течение Висконсинского оледенения и увеличения их ареалов в последующие периоды времени.

1Institute of Arctic Biology, University of Alaska, College, Alaska.

2Department of Wildlife Management, University of Alaska, College, Alaska. 


\section{INTRODUCTION}

The willow ptarmigan (Lagopus lagopus) is a circumpolar species that has been subdivided into 7 subspecies in North America (American Ornithologists Union 1957). Four subspecies occur in Alaska and adjacent Yukon Territory (Fig. 1). L. l. alascensis is distributed throughout Alaska except for the southern portions occupied by the following two subspecies: $L$. $l$. alexandrae on the Alaska Peninsula, Kenai Peninsula, and along a narrow margin of the Gulf of Alaska coast south into British Columbia, as far east as Atlin, and L. l. murei on Kodiak Island, the Shumagins and the Aleutians from Unimak westward (Gabrielson and Lincoln 1959). L. l. albus occurs from central Yukon Territory eastward and south to northern British Columbia (Fig. 1). Distinctions between the 4 subspecies are based primarily on summer plumage colour and width of the bill (Gabrielson and Lincoln 1959).

In our previous studies of willow ptarmigan, we noted that a sample of 1,206 individuals of the population occupying the central Brooks Range of northern Alaska formed statistically a single morphologically homogeneous population (West et al. 1968). In that study we compared measurements made by several experienced observers in this laboratory and found that variations from individual measurements did not alter the comparisons and that their records could be con-

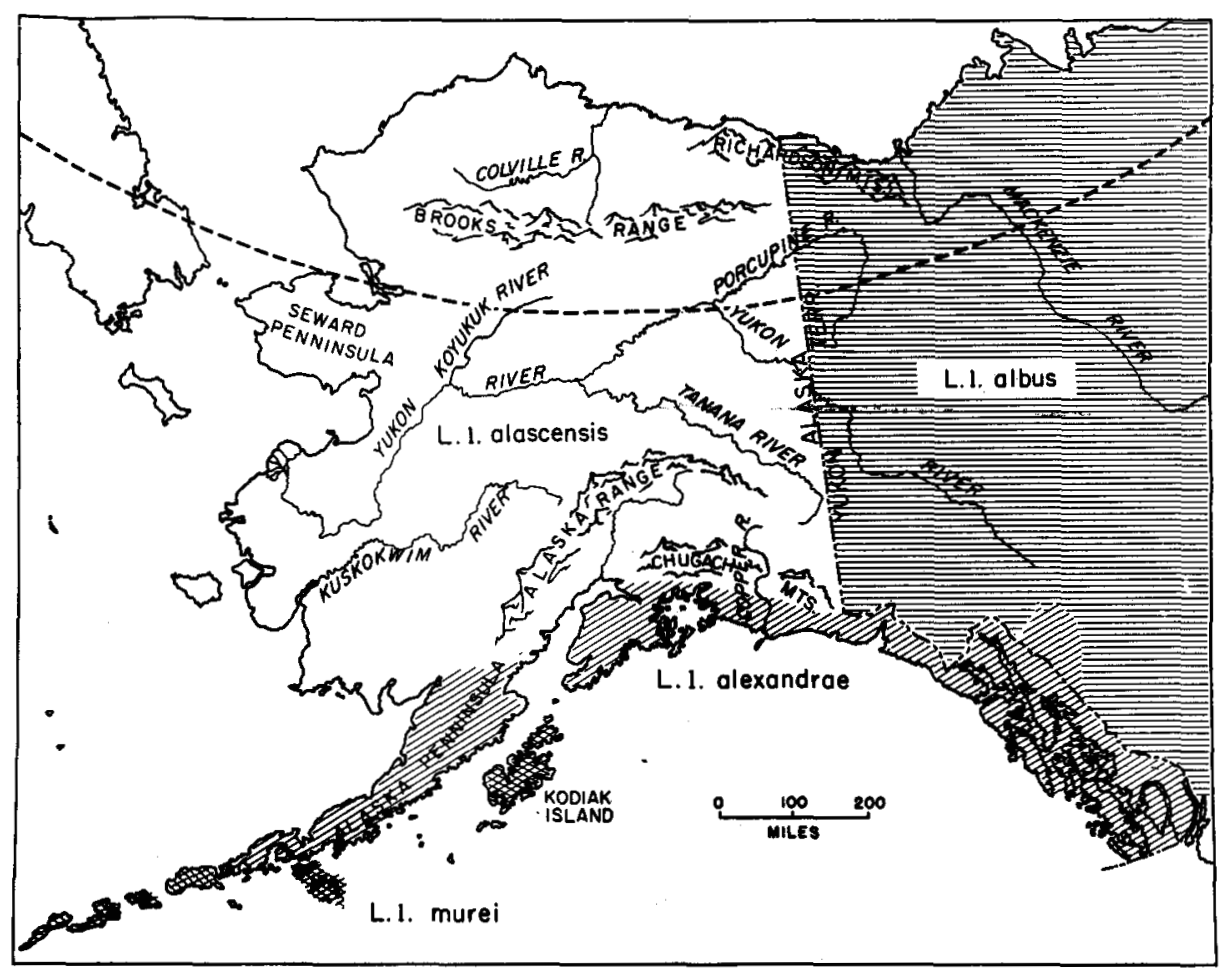

FIG. 1. Distribution of subspecies of willow ptarmigan according to A.O.U. (1957) and Gabrie]son and Lincoln (1959). 
sidered as one group. Therefore, we had a large population in northern Alaska to use as a basis of comparison for other populations throughout the State in order to see if the designations of the subspecies assigned on the basis of colour and size of bill corresponded with natural groupings of populations based on the dimensions of length and body weight.

Large numbers of ptarmigan are collected by residents for food and shot for sport. Even relatively small additions to these captures that we could obtain within lawful limits set for the normal protection of game provided over 2,600 specimens for measurement. Most of these samples were distributed in localities attributed to the subspecies $L$. $l$. alascensis which extends over 900 miles and 12 degrees of latitude from Barrow in the north to the southwestern coast. In this large geographical range we can see by statistically-valid numbers if dimensions of the birds of the subspecies vary or demonstrate clinal gradients.

At the margin of territories ascribed to $L$. l. alascensis we have had an opportunity to see if there are transitions in dimensions between the neighbouring populations $L$. l. alexandrae and $L . l$. albus. The extent of dimensional transitions and their relation to the location from which neighbouring samples were obtained could indicate if there were a clinal or geographical rate of exchange at the border of the subspecies.

\section{METHODS AND ANALYSIS}

Willow ptarmigan were collected by the authors and by resident native and non-native people throughout Alaska and a small portion of Yukon Territory (Table 1, Fig. 2). Obvious gaps in collecting localities occurred. This could have been due to a number of possibilities: the people contacted did not wish to collect for us (although a small remuneration was provided), there were no people in the area in question, there were no ptarmigan to collect, which was the case in the upper Kuskokwim area south of Middle Yukon (MDYU, Fig. 2). Ptarmigan were collected by shooting, primarily during the winter months (October through May), frozen in plastic bags and sent with attached collecting data to the Institute of Arctic Biology for processing. Each bird was thawed, weighed to the nearest gram, and the length of each wing (wrist to tip of longest primary of flattened wing) and length of the outer tail feather from point of insertion were measured by millimetre ruler. The bird was opened, the crop contents were removed and weighed and subtracted from the body weight to obtain the net body weight (weight-crop). Birds were aged by pigmentation of primary feathers (West et al. 1968) and sex was determined by examination of gonads. Many other characters were measured that will not be discussed here. Only those first year birds collected after 1 October were used in the analyses. Juvenile birds in this analysis are those from 3 to 14 months old and adults are those 15 months old or older.

The data were coded and punched on IBM cards for analysis. Cards were grouped by proximity of localities into a number of geographic units in order to obtain a sufficient sample size for each unit. Cards were then sorted by age and sex into 4 categories within each geographic unit (adult male, adult female, juvenile male, juvenile female) and frequency plots were made of wing and tail 


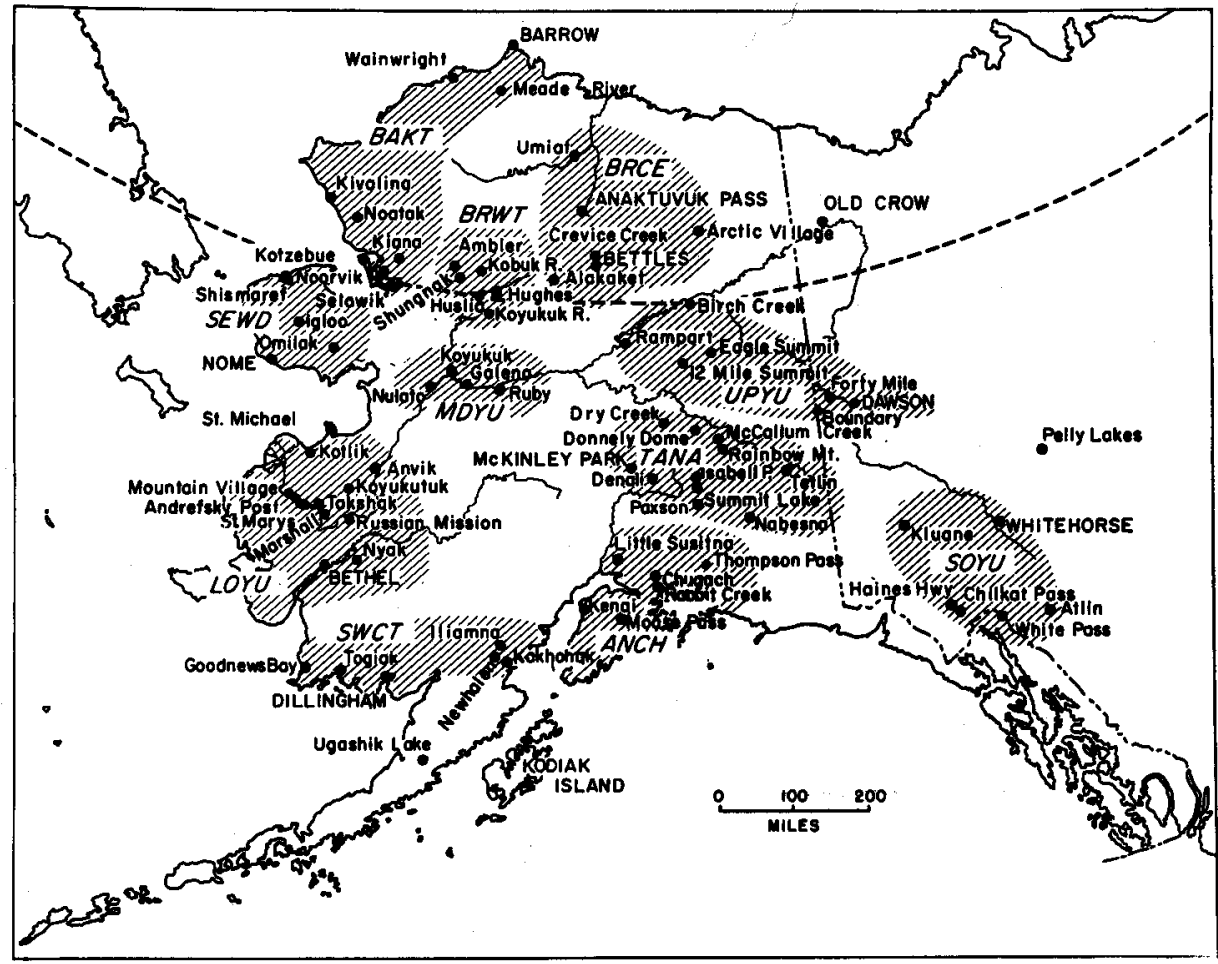

FIG. 2. Map of Alaska and adjacent Yukon Territory showing the collecting localities and analysis units of willow ptarmigan. (BRCE $=$ Brooks Range, central and east; BRWT $=$ Brooks Range, west; BAKT =Barrow to Kotzebue; SEWD =Seward Peninsula; LOYO=Lower Yukon Valley; SWCT =Southwest coast; MDYU=Middle Yukon Valley; UPYU=Upper Yukon Valley; TANA=Tanana Valley; $\mathrm{ANCH}=$ Anchorage; SOYU=Southern Yukon Territory.)

lengths. If the frequency distributions for wing and tail resulted in monophasic curves (judged by eye), the geographic unit was allowed to remain as a single analysis unit. However, if the curve appeared irregular, cards from individual collection localities at the periphery of the unit's geographic area were removed until the frequency curve appeared monophasic. The outliers were then joined to other groups occupying adjacent ranges. The result of this arbitrary but necessary analysis was a series of analysis units, each of which appeared by inspection to represent a homogeneous population on the basis of wing and tail measurements within the 4 age-sex categories (Table 1, Fig. 2).

Since total body weights may vary over 100 grams depending on the amount of food in the crop (Irving et al. 1967a), only net body weights (total weightcrop weight) are used in the following analyses. There are pronounced seasonal changes in net body weight, however (West and Meng 1968), and in order to make comparisons among populations, it was necessary to select a single time of year when body weights had been found to be stable for each group. Late winter, from February through April, was selected since this period showed the longest time interval with the least change in net body weight of all 4 age-sex categories of 
TABLE 1. Analysis units consisting of a group of adjacent geographic collecting localities that contain willow ptarmigan from morphologically homogeneous populations based on frequency distribution of wing and tail measurements.

\begin{tabular}{|c|c|}
\hline Analysis Unit & Locality (N. Latitude \& $W$. Longitude) \\
\hline Brooks Range central and east & $\begin{array}{l}\text { Umiat }\left(69^{\circ} 24^{\prime}-152^{\circ} 07^{\prime}\right) \\
\text { Anaktuvuk Pass }\left(68^{\circ} 09^{\prime}-151^{\circ} 46^{\prime}\right) \\
\text { Crevice Creek }\left(67^{\circ} 22^{\prime}-152^{\circ} 04^{\prime}\right) \\
\text { Bettles }\left(66^{\circ} 55^{\prime}-151^{\circ} 28^{\prime}\right) \\
\text { Allakaket }\left(66^{\circ} 34^{\prime}-152^{\circ} 38^{\prime}\right) \\
\text { Arctic Village }\left(68^{\circ} 10^{\prime}-145^{\circ} 40^{\prime}\right)\end{array}$ \\
\hline Brooks Range west & $\begin{array}{l}\text { Ambler }\left(67^{\circ} 10^{\prime}-158^{\circ} 31^{\prime}\right) \\
\text { Kobuk River }\left(66^{\circ} 55^{\prime}-156^{\circ} 55^{\prime}\right) \\
\text { Shungnak }\left(66^{\circ} 54^{\prime}-157^{\circ} 03^{\prime}\right) \\
\text { Hughes }\left(66^{\circ} 04^{\prime}-154^{\circ} 20^{\prime}\right) \\
\text { Huslia }\left(65^{\circ} 30^{\prime}-156^{\circ} 20^{\prime}\right) \\
\text { Koyukuk River }\left(65^{\circ} 40^{\prime}-156^{\circ} 25^{\prime}\right)\end{array}$ \\
\hline Barrow to Kotzebue & $\begin{array}{l}\text { Barrow }\left(71^{\circ} 20^{\prime}-156^{\circ} 55^{\prime}\right) \\
\text { Meade River }\left(70^{\circ} 32^{\prime}-157^{\circ} 02^{\prime}\right) \\
\text { Wainwright }\left(70^{\circ} 34^{\prime}-160^{\circ} 00^{\prime}\right) \\
\text { Kivalina }\left(67^{\circ} 44^{\prime}-164^{\circ} 40^{\prime}\right) \\
\text { Noatak }\left(67^{\circ} 33^{\prime}-162^{\circ} 56^{\prime}\right) \\
\text { Kiana }\left(66^{\circ} 58^{\prime}-160^{\circ} 28^{\prime}\right) \\
\text { Noorvik }\left(66^{\circ} 50^{\prime}-161^{\circ} 00^{\prime}\right) \\
\text { Selawik }\left(66^{\circ} 35^{\prime}-160^{\circ} 00^{\prime}\right) \\
\text { Kotzebue }\left(66^{\circ} 50^{\prime}-162^{\circ} 30^{\prime}\right)\end{array}$ \\
\hline Seward Peninsula & $\begin{array}{l}\text { Shishmaref }\left(66^{\circ} 12^{\prime}-166^{\circ} 08^{\prime}\right) \\
\text { Igloo }\left(64^{\circ} 58^{\prime}-165^{\circ} 04^{\prime}\right) \\
\text { Omilak }\left(65^{\circ} 03^{\prime}-162^{\circ} 40^{\prime}\right) \\
\text { Nome }\left(64^{\circ} 30^{\prime}-165^{\circ} 20^{\prime}\right)\end{array}$ \\
\hline Lower Yukon Valley & $\begin{array}{l}\text { St. Michael }\left(63^{\circ} 29^{\prime}-162^{\circ} 06^{\prime}\right) \\
\text { Kotlik }\left(63^{\circ} 02^{\prime}-163^{\circ} 33^{\prime}\right) \\
\text { Anvik }\left(62^{\circ} 38^{\prime}-160^{\circ} 12^{\prime}\right) \\
\text { Andreafsky Post }\left(62^{\circ} 03^{\prime}-163^{\circ} 10^{\prime}\right) \\
\text { Koyukutuk }\left(62^{\circ} 10^{\prime}-162^{\circ} 30^{\prime}\right) \\
\text { Mountain Village }\left(62^{\circ} 05^{\prime}-163^{\circ} 44^{\prime}\right) \\
\text { Takshak }\left(61^{\circ} 57^{\prime}-162^{\circ} 12^{\prime}\right) \\
\text { Marshall }\left(61^{\circ} 53^{\prime}-162^{\circ} 06^{\prime}\right) \\
\text { Russian Mission }\left(61^{\circ} 34^{\prime}-160^{\circ} 20^{\prime}\right) \\
\text { Nyac }\left(61^{\circ} 01^{\prime}-159^{\circ} 59^{\prime}\right) \\
\text { Bethel }\left(60^{\circ} 50^{\prime}-161^{\circ} 50^{\prime}\right)\end{array}$ \\
\hline Southwest Coast & $\begin{array}{l}\text { Goodnews Bay }\left(59^{\circ} 07^{\prime}-161^{\circ} 32^{\prime}\right) \\
\text { Togiak }\left(59^{\circ} 01^{\prime}-160^{\circ} 21^{\prime}\right) \\
\text { Dillingham }\left(59^{\circ} 10^{\prime}-158^{\circ} 28^{\prime}\right) \\
\text { Iliamna }\left(59^{\circ} 45^{\prime}-154^{\circ} 53^{\prime}\right) \\
\text { Newhalen }\left(59^{\circ} 42^{\prime}-154^{\circ} 57^{\prime}\right) \\
\text { Kokhonak }\left(59^{\circ} 26^{\prime}-154^{\circ} 25^{\prime}\right)\end{array}$ \\
\hline Middle Yukon Valley & $\begin{array}{l}\text { Koyukuk }\left(64^{\circ} 51^{\prime}-157^{\circ} 42^{\prime}\right) \\
\text { Galena }\left(64^{\circ} 45^{\prime}-156^{\circ} 55^{\prime}\right) \\
\text { Nulato }\left(64^{\circ} 45^{\prime}-158^{\circ} 05^{\prime}\right) \\
\text { Ruby }\left(64^{\circ} 45^{\prime}-155^{\circ} 28^{\prime}\right)\end{array}$ \\
\hline Upper Yukon Valley & $\begin{array}{l}\text { Birch Creek }\left(66^{\circ} 10^{\prime}-144^{\circ} 45^{\prime}\right) \\
\text { Rampart }\left(65^{\circ} 35^{\prime}-150^{\circ} 10^{\prime}\right) \\
\text { Dawson, Y.T. }\left(64^{\circ} 05^{\prime}-139^{\circ} 22^{\prime}\right) \\
\text { Eagle Summit }\left(65^{\circ} 38^{\prime}-145^{\circ} 30^{\prime}\right) \\
\text { Twelve-mile Summit }\left(65^{\circ} 30^{\prime}-145^{\circ} 35^{\prime}\right) \\
\text { Forty-mile River, Y.T. }\left(64^{\circ} 20^{\prime}-140^{\circ} 40^{\prime}\right) \\
\text { Boundary }\left(64^{\circ} 02^{\prime}-141^{\circ} 07^{\prime}\right)\end{array}$ \\
\hline
\end{tabular}




\begin{tabular}{|c|c|}
\hline Tanana Valley & $\begin{array}{l}\text { McKinley Park }\left(63^{\circ} 44^{\prime}-148^{\circ} 55^{\prime}\right) \\
\text { Donnelly Dome }\left(63^{\circ} 40^{\prime}-145^{\circ} 50^{\prime}\right) \\
\text { Dry Creek }\left(63^{\circ} 56^{\prime}-147^{\circ} 25^{\prime}\right) \\
\text { Denali }\left(63^{\circ} 10^{\prime}-147^{\circ} 30^{\prime}\right) \\
\text { McCallum Creek }\left(63^{\circ} 30^{\prime}-145^{\circ} 50^{\prime}\right) \\
\text { Rainbow Mountain }\left(63^{\circ} 12^{\prime}-145^{\circ} 30^{\prime}\right) \\
\text { Isabell Pass }\left(63^{\circ} 10^{\prime}-145^{\circ} 30^{\prime}\right) \\
\text { Summit Lake }\left(63^{\circ} 07^{\prime}-145^{\circ} 30^{\prime}\right) \\
\text { Paxson }\left(63^{\circ} 03^{\prime}-145^{\circ} 29^{\prime}\right) \\
\text { Nabesna }\left(62^{\circ} 23^{\prime}-143^{\circ} 02^{\prime}\right) \\
\text { Tetlin }\left(63^{\circ} 15^{\prime}-142^{\circ} 30^{\prime}\right)\end{array}$ \\
\hline Anchorage & $\begin{array}{l}\text { Chugach }\left(61^{\circ} 45^{\prime}-149^{\circ} 15^{\prime}\right) \\
\text { Little Susitna }\left(61^{\circ} 40^{\prime}-149^{\circ} 20^{\prime}\right) \\
\text { Rabbit Creek }\left(61^{\circ} 00^{\prime}-149^{\circ} 45^{\prime}\right) \\
\text { Thompson Pass }\left(61^{\circ} 00^{\prime}-145^{\circ} 50^{\prime}\right) \\
\text { Moose Pass }\left(60^{\circ} 29^{\prime}-149^{\circ} 22^{\prime}\right)\end{array}$ \\
\hline Southern Yukon Territory & $\begin{array}{l}\text { Chilkat Pass, Y.T. }\left(59^{\circ} 52^{\prime}-136^{\circ} 33^{\prime}\right) \\
\text { Haines Highway }\left(59^{\circ} 50^{\prime}-136^{\circ} 20^{\prime}\right) \\
\text { White Pass, Y.T. }\left(59^{\circ} 35^{\prime}-135^{\circ} 10^{\prime}\right) \\
\text { Atlin, B.C. }\left(59^{\circ} 40^{\prime}-133^{\circ} 25^{\prime}\right) \\
\text { Kluane, Y.T. }\left(61^{\circ} 00^{\prime}-138^{\circ} 20^{\prime}\right) \\
\text { Whitehorse, Y.T. }\left(60^{\circ} 40^{\prime}-135^{\circ} 15^{\prime}\right)\end{array}$ \\
\hline $\begin{array}{l}\text { Miscellaneous } \\
\text { Alaska }\end{array}$ & $\begin{array}{l}\text { Kodiak Island }\left(57^{\circ} 50^{\prime}-152^{\circ} 30^{\prime}\right) \\
\text { Ugashik Lake }\left(57^{\circ} 30^{\prime}-157^{\circ} 00^{\prime}\right) \\
\text { Kenai }\left(60^{\circ} 35^{\prime}-151^{\circ} 16^{\prime}\right)\end{array}$ \\
\hline Yukon Territory & $\begin{array}{l}\text { Old Crow }\left(67^{\circ} 34^{\prime}-139^{\circ} 53^{\prime}\right) \\
\text { Pelley Lakes }\left(62^{\circ} 00^{\prime}-128^{\circ} 30^{\prime}\right)\end{array}$ \\
\hline
\end{tabular}

Brooks Range ptarmigan (West and Meng 1968). It was assumed that at this time of year, when no extra energy-demanding activities other than winter existence occurred such as molt or breeding (West 1968) net body weight would be uniformly stable in all populations under investigation. Owing to the small sample sizes in many analysis units, it was necessary to choose more than one month for comparisons. Therefore the analysis groups for net body weight contained only those individuals collected from February through April.

We noted earlier that a combination of wing plus tail length gave a better indication of sex than either measurement alone - at least in adult ptarmigan (West et al. 1968). Therefore we analysed our population samples by this character in addition to the preceding three and compared the analysis units using the factors of wing length, tail length, wing plus tail lengths, and net body weight (February to April).

Each sex and age category was compared with the corresponding category in each analysis unit by the use of Duncan's multiple range test for unequal sample sizes and heteroscedastic means (Duncan 1957) modified by using Newman and Keul's tables of critical values (Miller 1966). A probability level of 0.01 was used to test for significance of sample separations.

\section{RESULTS AND DISCUSSION}

The sample sizes, means and standard deviations for each of the characteristics used in the comparisons are listed by age-sex category in Tables 2 to 5 . There is 
TABLE 2. Means and standard deviations of wing, tail and body weight measurements of adult male willow ptarmigan grouped into analysis units of different geographical areas.

\begin{tabular}{|c|c|c|c|c|c|c|c|c|}
\hline ADULT MALES & $\mathbf{n}$ & $\begin{array}{l}\text { Wing Length } \\
\overline{\mathbf{x}} \pm \text { S.D. (mm) }\end{array}$ & $\mathbf{n}$ & $\begin{array}{l}\text { Tail Length } \\
\bar{x} \pm S . D .(m m)\end{array}$ & $\underset{\mathbf{n}}{\text { Win }}$ & $\begin{array}{l}g+\text { Tail Length } \\
\overrightarrow{\mathbf{x}} \pm \text { S.D. (mm) }\end{array}$ & $\underset{\mathrm{n}}{\mathrm{Ne}}$ & $\begin{array}{l}\text { Body Weight* } \\
\overline{\mathbf{x}} \pm \text { S.D. }(\mathrm{g})\end{array}$ \\
\hline $\begin{array}{l}\text { Brooks Range, } \\
\text { central and east } \\
\text { Brooks Range, west } \\
\text { Barrow to Kotzebue } \\
\text { Seward Peninsula } \\
\text { Lower Yukon Valley } \\
\text { Southwest Coast } \\
\text { Middle Yukon Valley } \\
\text { Upper Yukon Valley } \\
\text { Tanana Valley } \\
\text { Southern Yukon T. }\end{array}$ & $\begin{array}{r}395 \\
35 \\
58 \\
41 \\
87 \\
58 \\
18 \\
9 \\
76 \\
14\end{array}$ & $\begin{array}{l}205.47 \pm 4.75 \\
203.43 \pm 3.65 \\
204.50 \pm 4.72 \\
203.39 \pm 4.51 \\
204.79 \pm 5.92 \\
203.88 \pm 4.63 \\
204.89 \pm 4.58 \\
200.78 \pm 5.17 \\
195.33 \pm 5.16 \\
192.36 \pm 4.75\end{array}$ & $\begin{array}{r}366 \\
26 \\
58 \\
42 \\
82 \\
61 \\
16 \\
12 \\
58 \\
14\end{array}$ & $\begin{array}{l}122.57 \pm 5.34 \\
123.31 \pm 5.35 \\
124.55 \pm 5.58 \\
122.69 \pm 3.74 \\
125.21 \pm 5.71 \\
123.44 \pm 5.25 \\
125.69 \pm 4.57 \\
123.25 \pm 4.65 \\
118.88 \pm 5.03 \\
119.93 \pm 3.97\end{array}$ & $\begin{array}{r}360 \\
26 \\
53 \\
40 \\
81 \\
56 \\
16 \\
9 \\
58 \\
14\end{array}$ & $\begin{array}{l}328.20 \pm 8.73 \\
327.12 \pm 8.12 \\
329.36 \pm 9.18 \\
326.03 \pm 7.33 \\
330.26 \pm 9.89 \\
327.00 \pm 8.71 \\
330.63 \pm 8.45 \\
324.56 \pm 7.81 \\
313.47 \pm 9.06 \\
312.24 \pm 7.74\end{array}$ & $\begin{array}{r}222 \\
22 \\
17 \\
12 \\
66 \\
38 \\
17 \\
9 \\
94 \\
1\end{array}$ & $\begin{array}{l}612.73 \pm 38.65 \\
587.17 \pm 41.52 \\
626.92 \pm 36.46 \\
643.03 \pm 51.34 \\
626.32 \pm 42.99 \\
615.69 \pm 37.75 \\
596.98 \pm 33.32 \\
576.93 \pm 39.18 \\
546.55 \pm 42.42 \\
545\end{array}$ \\
\hline
\end{tabular}

*Months of Feb., March and April only

TABLE 3. Means and standard deviations of wing, tail and body weight measurements of adult female willow ptarmigan grouped into analysis units of different geographical areas.

\begin{tabular}{|c|c|c|c|c|c|c|c|c|}
\hline ADULT FEMALES & $\mathbf{n}$ & $\begin{array}{l}\text { Ning Length } \\
\overline{\mathbf{x}} \pm \text { S.D. (mm) }\end{array}$ & $\mathbf{n}$ & $\begin{array}{l}\text { Tail Length } \\
\overline{\mathbf{x}} \pm \text { S.D. }(\mathrm{mm})\end{array}$ & $\underset{\mathrm{n}}{\mathbf{W i n}}$ & $\begin{array}{l}\mathrm{g}+\text { Tail Length } \\
\overline{\mathbf{x}} \pm \text { S.D. (mm) }\end{array}$ & & $\begin{array}{l}\text { Body Weight* } \\
\bar{x} \pm \text { S.D. (g) }\end{array}$ \\
\hline $\begin{array}{l}\text { Brooks Range, } \\
\text { central and east } \\
\text { Brooks Range, west } \\
\text { Barrow to Kotzebue } \\
\text { Seward Peninsula } \\
\text { Lower Yukon Valley } \\
\text { Southwest Coast } \\
\text { Middle Yukon Valley } \\
\text { Upper Yukon Valley } \\
\text { Tanana Valley } \\
\text { Southern Yukon T. }\end{array}$ & $\begin{array}{r}171 \\
35 \\
24 \\
8 \\
60 \\
55 \\
36 \\
11 \\
60 \\
26\end{array}$ & $\begin{array}{l}192.09 \pm 4.24 \\
191.69 \pm 4.14 \\
190.86 \pm 5.61 \\
187.50 \pm 3.21 \\
193.77 \pm 4.93 \\
191.56 \pm 4.70 \\
191.58 \pm 4.20 \\
189.36 \pm 6.80 \\
184.60 \pm 4.81 \\
180.77 \pm 4.62\end{array}$ & $\begin{array}{r}157 \\
28 \\
23 \\
6 \\
58 \\
55 \\
33 \\
14 \\
45 \\
28\end{array}$ & $\begin{array}{l}110.65 \pm 4.93 \\
111.71 \pm 4.09 \\
110.74 \pm 3.92 \\
110.17 \pm 4.36 \\
112.66 \pm 4.72 \\
112.56 \pm 4.00 \\
112.49 \pm 4.33 \\
109.93 \pm 5.64 \\
108.69 \pm 4.49 \\
109.11 \pm 3.26\end{array}$ & $\begin{array}{r}150 \\
27 \\
21 \\
4 \\
56 \\
54 \\
33 \\
7 \\
45 \\
26\end{array}$ & $\begin{array}{l}302.96 \pm 7.52 \\
303.78 \pm 6.66 \\
300.81 \pm 8.89 \\
298.50 \pm 7.72 \\
306.18 \pm 8.74 \\
304.43 \pm 7.47 \\
304.24 \pm 7.81 \\
296.57 \pm 14.37 \\
292.96 \pm 8.47 \\
290.04 \pm 7.52\end{array}$ & $\begin{array}{r}75 \\
25 \\
16 \\
48 \\
37 \\
28 \\
16 \\
79 \\
2\end{array}$ & $\begin{array}{l}524.82 \pm 35.92 \\
523.58 \pm 39.68 \\
526.00 \pm 36.39 \\
544.94 \pm 33.62 \\
531.14 \pm 36.35 \\
532.53 \pm 30.34 \\
512.10 \pm 32.48 \\
473.55 \pm 27.19 \\
472.4\end{array}$ \\
\hline
\end{tabular}

*Months of Feb., March and April only

TABLE 4. Means and standard deviations of wing, tail and body weight measurements of juvenile male willow ptarmigan grouped into analysis units of different geographical areas.

\begin{tabular}{|c|c|c|c|c|c|c|c|c|}
\hline JUVENILE MALES & $\mathbf{n}$ & $\begin{array}{l}\text { Wing Length } \\
\bar{x} \pm \text { S.D. (mm) }\end{array}$ & $\mathbf{n}$ & $\begin{array}{l}\text { Tail Length } \\
\overline{\mathbf{x}} \pm \text { S.D. }(\mathrm{mm})\end{array}$ & $\underset{\mathbf{n}}{\mathbf{W i}}$ & $\begin{array}{l}+ \text { Tail Le } \\
\overline{\mathrm{x}} \pm \text { S.D. }\end{array}$ & & $\begin{array}{l}\text { Body Weight* } \\
\bar{x} \pm \text { S.D. (g) }\end{array}$ \\
\hline $\begin{array}{l}\text { Brooks Range, } \\
\text { central and east } \\
\text { Brooks Range, west } \\
\text { Barrow to Kotzebue } \\
\text { Seward Peninsula } \\
\text { Lower Yukon Valley } \\
\text { Southwest Coast } \\
\text { Middle Yukon Valley } \\
\text { Upper Yukon Valley } \\
\text { Tanana Valley } \\
\text { Southern Yukon T. }\end{array}$ & $\begin{array}{r}339 \\
49 \\
44 \\
45 \\
48 \\
45 \\
23 \\
1 \\
27 \\
3\end{array}$ & $\begin{array}{l}201.62 \pm 5.17 \\
200.22 \pm 5.91 \\
203.16 \pm 4.89 \\
199.93 \pm 4.51 \\
203.38 \pm 5.00 \\
200.39 \pm 3.89 \\
199.70 \pm 4.19 \\
197 \\
193.56 \pm 5.92 \\
188.67\end{array}$ & $\begin{array}{r}272 \\
34 \\
41 \\
48 \\
42 \\
46 \\
18 \\
19 \\
2\end{array}$ & $\begin{array}{l}117.22 \pm 5.30 \\
119.27 \pm 3.73 \\
119.98 \pm 5.13 \\
121.00 \pm 3.57 \\
121.55 \pm 5.07 \\
119.44 \pm 4.62 \\
119.68 \pm 3.53 \\
117.63 \pm 7.72 \\
113.5\end{array}$ & $\begin{array}{r}264 \\
32 \\
40 \\
44 \\
41 \\
45 \\
18 \\
15 \\
2\end{array}$ & $\begin{array}{l}318.53 \pm 9.41 \\
320.34 \pm 7.64 \\
323.23 \pm 9.33 \\
321.14 \pm 7.03 \\
324.59 \pm 8.94 \\
319.73 \pm 7.28 \\
319.44 \pm 6.59 \\
314.00 \pm 12.02 \\
302.5\end{array}$ & $\begin{array}{r}144 \\
20 \\
26 \\
3 \\
32 \\
45 \\
22 \\
2 \\
32 \\
1\end{array}$ & $\begin{array}{l}586.65 \pm 44.86 \\
572.77 \pm 32.68 \\
615.09 \pm 42.37 \\
593.6 \\
608.53 \pm 37.63 \\
591.91 \pm 35.80 \\
571.98 \pm 29.07 \\
539.4 \\
523.41 \pm 44.22 \\
466\end{array}$ \\
\hline
\end{tabular}

*Months of Feb., March and April only 
TABLE 5. Means and standard deviations of wing, tail and body weight measurements of juvenile female willow ptarmigan grouped into analysis units of different geographical areas.

\begin{tabular}{|c|c|c|c|c|c|c|c|c|}
\hline JUVENILE FEMALES & \multicolumn{2}{|r|}{$\begin{array}{l}\text { Wing Length } \\
\bar{x} \pm \text { S.D. (mm) }\end{array}$} & 几 & $\begin{array}{l}\text { Tail Length } \\
\bar{x} \pm \text { S.D. }(\mathrm{mm})\end{array}$ & \multicolumn{2}{|c|}{$\begin{array}{c}\text { Wing }+ \text { Tail Length } \\
n\end{array}$} & \multicolumn{2}{|c|}{$\begin{array}{l}\text { Net Body Weight } \\
n \quad \bar{x} \pm \text { S.D. }(\mathrm{g})\end{array}$} \\
\hline $\begin{array}{l}\text { Brooks Range, } \\
\text { central and east } \\
\text { Brooks Range, west } \\
\text { Barrow to Kotzebue } \\
\text { Seward Peninsula } \\
\text { Lower Yukon Valley } \\
\text { Southwest Coast } \\
\text { Middle Yukon Valley } \\
\text { Upper Yukon Valley } \\
\text { Tanana Valley } \\
\text { Southern Yukon T. }\end{array}$ & $\begin{array}{r}243 \\
56 \\
26 \\
11 \\
55 \\
39 \\
63 \\
14 \\
14 \\
5\end{array}$ & $\begin{array}{l}190.04 \pm 4.93 \\
189.27 \pm 5.06 \\
192.81 \pm 4.43 \\
187.91 \pm 4.25 \\
191.71 \pm 4.64 \\
189.33 \pm 4.83 \\
189.57 \pm 4.57 \\
188.07 \pm 4.98 \\
184.29 \pm 3.38 \\
178.66 \pm 5.55\end{array}$ & $\begin{array}{r}221 \\
38 \\
24 \\
10 \\
48 \\
39 \\
63 \\
10 \\
11 \\
4\end{array}$ & $\begin{array}{l}107.39 \pm 4.95 \\
109.00 \pm 4.21 \\
110.50 \pm 4.15 \\
108.40 \pm 5.38 \\
112.15 \pm 3.33 \\
110.56 \pm 3.86 \\
110.27 \pm 3.70 \\
109.70 \pm 4.72 \\
109.09 \pm 4.70 \\
108.00 \pm 3.16\end{array}$ & $\begin{array}{r}211 \\
37 \\
24 \\
10 \\
48 \\
37 \\
59 \\
8 \\
9 \\
4\end{array}$ & $\begin{array}{l}297.60 \pm 8.82 \\
298.76 \pm 8.75 \\
302.96 \pm 7.18 \\
296.10 \pm 8.57 \\
303.48 \pm 6.33 \\
299.97 \pm 7.76 \\
299.64 \pm 7.10 \\
298.75 \pm 8.33 \\
294.89 \pm 5.93\end{array}$ & $\begin{array}{r}106 \\
35 \\
15 \\
1 \\
30 \\
27 \\
59 \\
2 \\
13 \\
-\end{array}$ & $\begin{array}{l}504.75 \pm 38.46 \\
503.28 \pm 28.64 \\
538.71 \pm 35.68 \\
516 \\
536.16 \pm 31.92 \\
508.35 \pm 24.71 \\
504.51 \pm 33.66 \\
506.7 \\
461.11 \pm 38.39\end{array}$ \\
\hline
\end{tabular}

*Months of Feb., March and April only

considerable variation in sample size among analysis units for any one character and also some variation in sample size within each analysis unit for all characters. The former is explained simply on the basis of availability of specimens and the grouping of specimens into analysis units. The latter is explained by the fact that early in the study not all characters were determined on each specimen or else the character was not suitable for determination - i.e. bird badly shot, wing and/or tail feathers missing, etc. Only analysis units with a sample size of 10 or more measurements in each age-sex eategory were used in the multiple range test comparisons.

The results of the multiple range test are shown in Table 6. Lines under the analysis units on the same horizontal plane indicate homogeneous subsets of data that are significantly different at the 0.01 per cent confidence level from other underlined subsets. Therefore in Table 6, the wing lengths of adult males from the first two analysis units, Southern Yukon Territory and Tanana, are not significantly different from each other but are significantly different from all of the rest of the analysis units. Likewise none of the remaining units from Upper Yukon to Brooks Range, central and east, is significantly different from the other.

Owing to large variations in sample size, unexpected anomalies sometimes occurred in this analysis. For example in inspecting the results of tail lengths of adult males (Table 6), we see that the second line shows a homogeneous subset including Southern Yukon Territory through Barrow to Kotzebue which skips over Lower Yukon Valley but includes Middle Yukon Valley. At the same time the third line indicates another subset which includes most of the other units and Lower Yukon Valley. In checking Table 2, we see that Lower Yukon has a sample size of 82 and Middle Yukon Valley only 16 . The decrease in variance as a result of the large sample size has eliminated Lower Yukon Valley from the second subset which includes Brooks Range, central and east with a sample size of 366 . In the third subset, Brooks Range, central and east is not included and therefore Lower and Middle Yukon Valley are included in the same subset.

The pattern in forming homogeneous subsets among the 4 age-sex categories was not always consistent. For adult males, Brooks Range, central and east birds have the longest wings, but are lighter in weight than Seward Peninsula birds (by 30 grams). For adult females, birds from Lower Yukon Valley are the largest in 
TABLE 6. Multiple range test comparisons of analysis units of willow ptarmigan arranged in order by means of length or weight. Dotted lines under units indicate homogeneous subsets as determined by multiple range tests at the 0.01 level of significance.

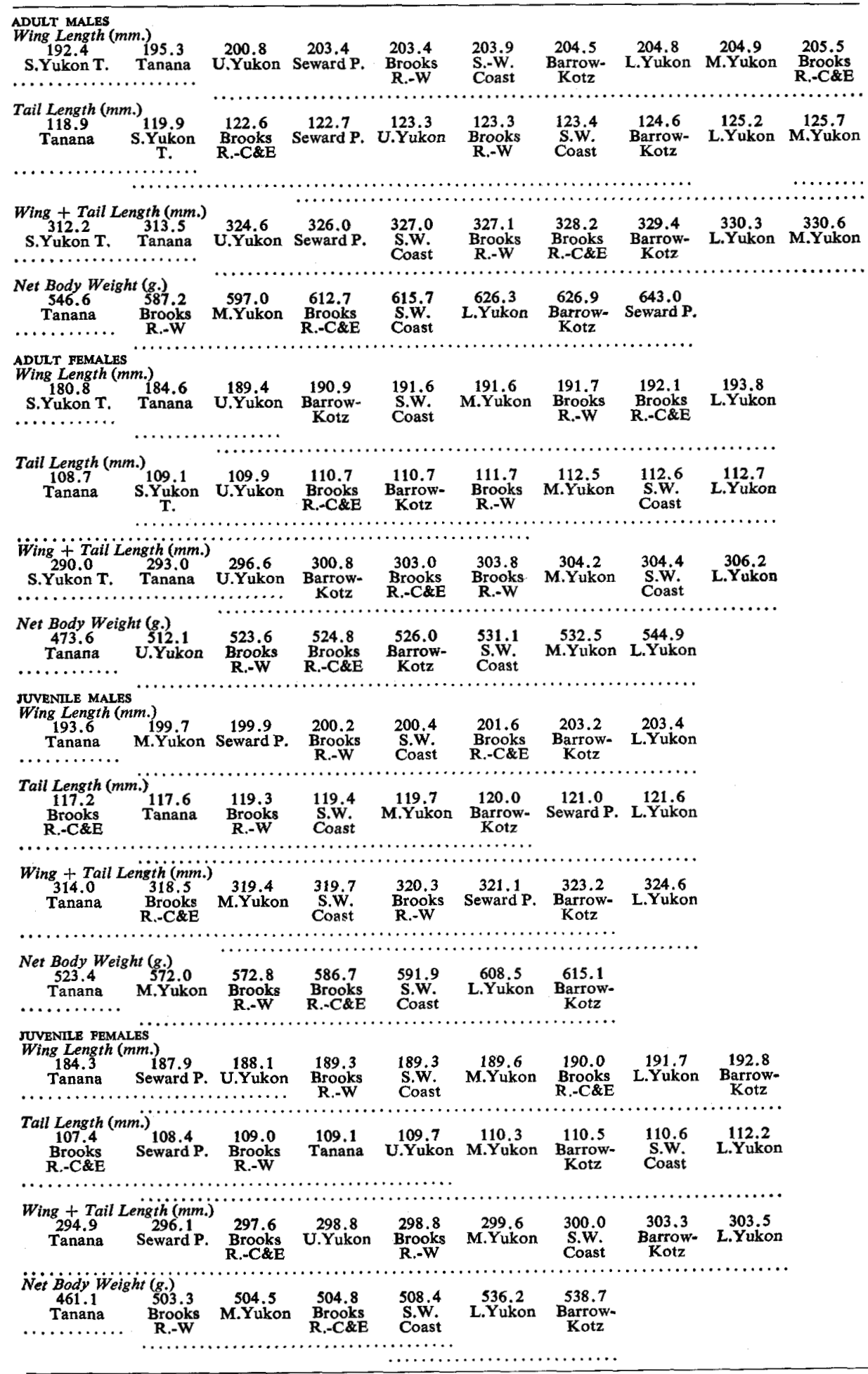


all characters measured. Lower Yukon Valley also contains the longest winged and tailed juvenile males but those from Barrow to Kotzebue are 7 grams heavier than their Lower Yukon Valley relatives. And for juvenile females the two groups, Lower Yukon Valley and Barrow to Kotzebue, far outweigh their neighbours (by 30 grams) but the linear dimensions differ little from those of other analysis units.

Large and consistent differences did occur between some subsets and these indicate that in the great majority of cases, Tanana birds are smaller in terms of length and body weight than birds to the north, west, or southwest of them in Alaska (Fig. 2). There is a strong indication that birds of Southern Yukon Territory are even smaller in dimension and weight than the Tanana Valley birds. Unfortunately, we did not have a sufficient sample for testing in all cases, but by referring to Tables 2-5, one can note that the means of birds of Southern Yukon Territory fall below those of the Tanana Valley in almost every case. Birds from Upper Yukon Valley are borderline and if we had enough specimens, we believe the group could be split placing the birds from Forty-mile, Dawson, and Boundary in with the Tanana Valley group (Fig. 2).

The miscellaneous groups of birds listed in Table 7 may also be compared with Table 6. Starting with the northern part of the range we note that the few birds from Old Crow in arctic Yukon Territory are larger than any group listed in Table 6, and are probably more closely associated with the Brooks Range population than with the smaller Upper Yukon birds. Birds from Pelley Lake at the border of Yukon and Northwest Territories, however, are decidedly smaller, being similar in dimension and weight to birds from Tanana and Southern Yukon.

The birds from Anchorage and Kenai are small, with means of length and weight falling in the range of or below those from Southern Yukon Territory and Tanana, and therefore are distinct from their neighbours to the west (Southwest Coast). The few birds from Ugashik Lake on the Alaska Peninsula are medium to large and may be related to those in the Southwest Coast analysis unit.

TABLE 7. Means and standard deviations of wing, tail, and body weight measurements of willow ptarmigan from locations not included in Tables 2 to 5 .

\begin{tabular}{|c|c|c|c|c|c|c|c|c|}
\hline & n & $\begin{array}{l}\text { Wing Length } \\
\overline{\mathbf{x}} \pm \text { S.D. (mm) }\end{array}$ & $\mathbf{n}$ & $\begin{array}{l}\text { Tail Length } \\
\overline{\mathbf{x}} \pm \text { S.D. }(\mathrm{mm})\end{array}$ & $\underset{\mathbf{n}}{\text { Wing }}$ & $\begin{array}{c}g+\text { Tail Length } \\
\bar{x} \pm \text { S.D. }(\mathrm{mm})\end{array}$ & $\mathrm{Ne}_{\mathrm{n}}$ & $\begin{array}{l}\text { Body Weight } \\
\bar{x} \pm \text { S.D. (g) }\end{array}$ \\
\hline $\begin{array}{l}\text { Adult Males } \\
\text { Pelley Lakes } \\
\text { Anchorage } \\
\text { Ugashik Lake } \\
\text { Kodiak Island }\end{array}$ & $\begin{array}{l}3 \\
9 \\
5 \\
6\end{array}$ & $\begin{array}{l}191.67 \\
193.33 \pm 3.64 \\
203.00 \pm 9.57 \\
206.50 \pm 5.54\end{array}$ & $\begin{array}{l}3 \\
9 \\
3 \\
7\end{array}$ & $\begin{array}{l}116.33 \\
117.78 \pm 5.09 \\
122.00 \\
124.29 \pm 4.79\end{array}$ & $\begin{array}{l}3 \\
8 \\
3 \\
6\end{array}$ & $\begin{array}{l}308.00 \\
310.75 \pm 7.65 \\
338.00 \\
331.50 \pm 9.18\end{array}$ & $\frac{3}{11}$ & $\begin{array}{l}492.43 \\
610.67 \pm 30.16\end{array}$ \\
\hline $\begin{array}{l}\text { Adult Females } \\
\text { Old Crow } \\
\text { Pelley Lakes } \\
\text { Anchorage } \\
\text { Kenai } \\
\text { Kodiak Island }\end{array}$ & $\begin{array}{l}\mathbf{4} \\
\mathbf{3} \\
\mathbf{3} \\
\mathbf{2} \\
\mathbf{7}\end{array}$ & $\begin{array}{l}196.25=0.96 \\
181.33 \\
180.67 \\
181.5 \\
192.43 \pm 3.60\end{array}$ & $\begin{array}{l}4 \\
3 \\
5 \\
7\end{array}$ & $\begin{array}{l}118.50 \pm 3.51 \\
103.00 \\
105.20 \pm 10.35 \\
111.71 \pm 3.25\end{array}$ & $\begin{array}{l}4 \\
3 \\
2 \\
6\end{array}$ & $\begin{array}{l}314.75 \pm 3.50 \\
284.33 \\
283.5 \\
303.83 \pm 6.08\end{array}$ & $\begin{array}{r}4 \\
3 \\
4 \\
13\end{array}$ & $\begin{array}{l}612.73 \pm 46.89 \\
422.33 \\
494.28 \pm 15.83 \\
531.68 \pm 22.45\end{array}$ \\
\hline $\begin{array}{l}\text { Juvenile Males } \\
\text { Old Crow } \\
\text { Anchorage } \\
\text { Kenai } \\
\text { Ugashik Lake } \\
\text { Kodiak Island }\end{array}$ & $\begin{array}{r}1 \\
6 \\
2 \\
3 \\
10\end{array}$ & $\begin{array}{l}208 \\
189.50 \pm 8.55 \\
190.5 \\
205.33 \\
199.80 \pm 5.16\end{array}$ & $\frac{1}{8}$ & $\begin{array}{l}125 \\
106.00 \pm 8.91 \\
119.5 \\
116.64 \pm 4.27\end{array}$ & $\frac{1}{6}$ & $\begin{array}{l}333 \\
293.33 \pm 14.83 \\
322.5 \\
314.50 \pm 8.12\end{array}$ & $\frac{1}{\frac{1}{17}}$ & $\begin{array}{l}615 \\
512.68\end{array}$ \\
\hline $\begin{array}{l}\text { Juvenile Females } \\
\text { Old Crow } \\
\text { Anchorage } \\
\text { Kodiak Island }\end{array}$ & $\begin{array}{r}2 \\
2 \\
11\end{array}$ & $\begin{array}{l}193.5 \\
182.5 \\
186.45 \pm 3.72\end{array}$ & $\begin{array}{r}1 \\
2 \\
11\end{array}$ & $\begin{array}{l}110 \\
106,5 \\
105,64 \pm 4.92\end{array}$ & $\begin{array}{r}1 \\
2 \\
10\end{array}$ & $\begin{array}{l}297 \\
289.0 \\
292.60 \pm 6.67\end{array}$ & $\begin{array}{r}2 \\
2 \\
28\end{array}$ & $\begin{array}{l}588.1 \\
446.4 \\
508.42 \pm 36.10\end{array}$ \\
\hline
\end{tabular}

*Months of Feb., March and April only 
Birds from Kodiak Island appear to be distinct from others already considered. Both adult males and females have very long wings, medium to long tails and medium body weights. Juveniles, on the other hand, have relatively short wings and tails and medium body weights when compared to the data in Table 6.

On the basis of the comparisons given in Table 6 and those just discussed, we can postulate that most of Alaska is populated by the Alaska willow ptarmigan L. l. alascensis, its range being the largest of the subspecies in Alaska, extending at least from Old Crow on the Porcupine River westward and northward to the Arctic Coast, from there throughout the Brooks Range and Seward Peninsula, south through the middle and upper Yukon Valley to Bristol Bay and the base of the Alaska Peninsula (Fig. 3). In our sample, a smaller population, probably $L$. $l$. albus, occurs in the Tanana-Yukon uplands, and along the upper Yukon River in Yukon Territory east to Pelley Lake and south to the headwaters of the Yukon and mountain passes of southern Yukon Territory (Fig. 3).

Based on the criteria of size and weight above, Anchorage birds could also belong to $L$. l. albus, but since they are separated by the Chugach Mountains from the populations attributed to $L$. l. albus, they probably belong to $L$. l. alexandrae as do those specimens from the Kenai Peninsula (Fig. 3). Our sample from Southern Yukon Territory in the vicinity of Atlin is from near the border ascribed to the

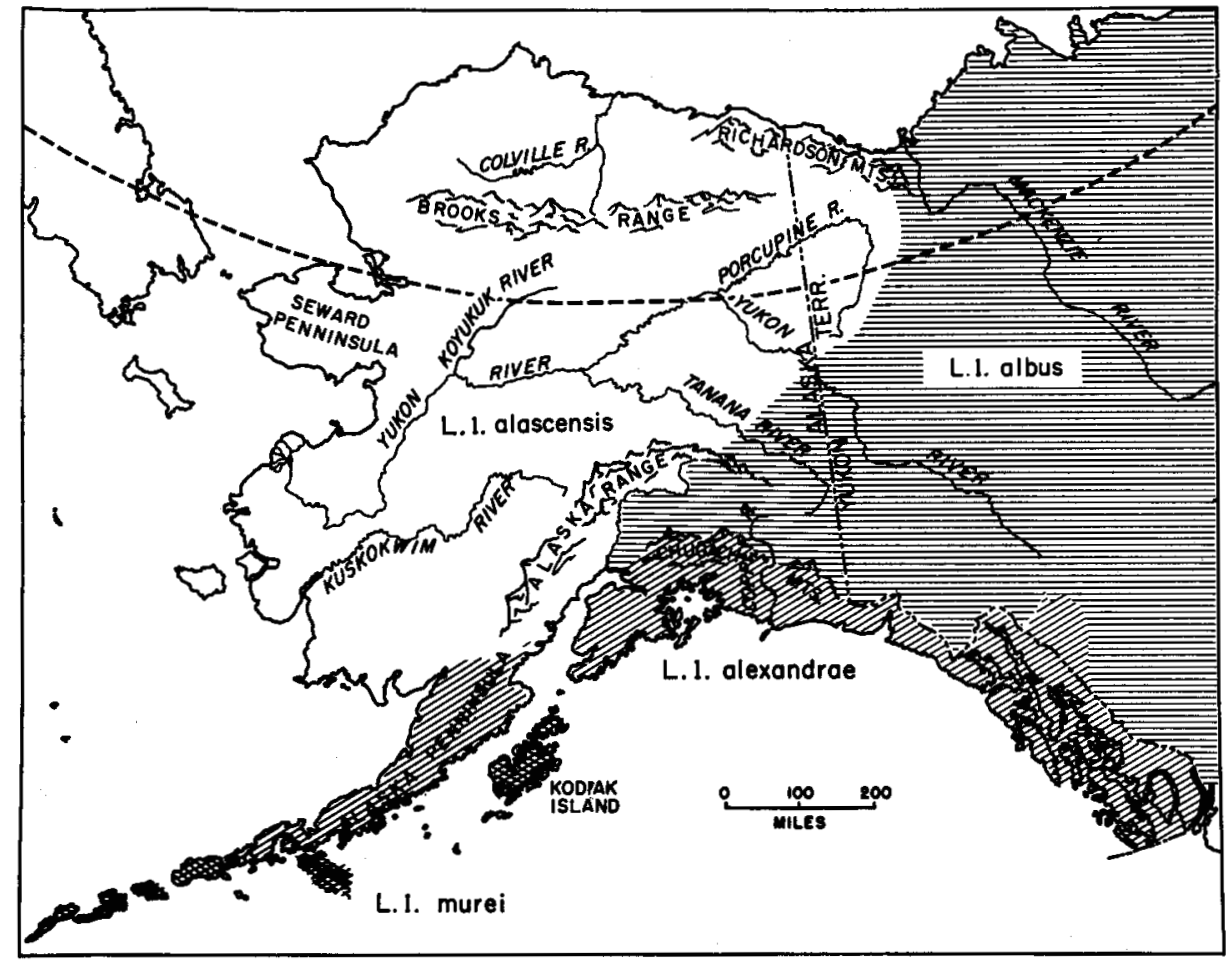

FIG. 3. Proposed distribution of 4 subspecies of willow ptarmigan in Alaska and adjacent Yukon Territory based on body size and weight measurements. 
meeting of $L$. l. albus of the interior with coastal $L$. $l$. alexandrae which are not distinguishable by the dimensions we have compiled.

The ptarmigan from the Southwest Coast (Table 1) include birds from the northern side of Bristol Bay along with examples from Iliamna, Newhalen and Kokhonak at the base of the Alaska Peninsula. In dimensions these ptarmigan conform with L. l. alascensis (Tables 2 to 6). A small sample (8) from Ugashik Lake, further west and north of the mountainous spine of the Peninsula, also conforms with $L$. $l$. alascensis. Since their dimensions are distinct from those of $L$. $l$. alexandrae, we suggest that in winter, when our examples were taken, the base and part of the northern side of the Peninsula were occupied by $L$. $l$. alascensis.

The ranges conventionally ascribed to willow ptarmigan can be well established by the plumage of birds taken in summer and also, though less definitely, by size of bill. In the central Brooks Range the annual range of willow ptarmigan extends by migrations over several hundred miles, and their southern limit in winter is more than a hundred miles from the more northern centre of nesting on tundra in summer. Without knowledge of annual local migrations, boundaries between populations are not closely definable. It would appear that on the Alaska Peninsula the two subspecies, $L$. $l$. alascensis and $L$. $l$. alexandrae may nearly intermingle but that $L$. $l$. alascensis nevertheless retains dimensional distinction by resorting to its own summer nesting areas. In the extensive longitudinal range ascribed to $L . l$. murei it is not indicated that willow ptarmigan are differentiated as is $L$. mutus, for which 8 subspecies are named over the Aleutian Islands (A.O.U. 1957).

In comparing Fig. 1, showing the range of the 4 subspecies based on summer plumage and bill shape, with Fig. 3 showing the range based on wing and tail lengths and body weights in winter, we see that in the north the range of $L$. $l$. alascensis extends eastward into Yukon Territory, at least to Old Crow and perhaps to the Richardson Mountains. Although Gabrielson and Lincoln (1959) include the upper Copper River Valley in the range of alascensis, by their dimensions the smaller birds in this area and the upper Tanana River drainage belong to L. l. albus, separated from $L$. l. alexandrae at this point by the Chugach Mountains (Fig. 3).

The standard deviations of wing and tail length of $L$. $l$. alascensis differ surprisingly little among localities and are of the same order of magnitude as those within the largest sample (Brooks Range, central and east; Tables 2 to 5). Since we have already shown the Brooks Range population to be morphologically homogeneous on the basis of wing and tail length measurements (West et al. 1968) it is reasonable to assume that each of the other groups with fewer examples and the same standard deviation (and exhibiting a monophasic non-skewed distribution) is also homogeneous. The subspecies $L$. $l$. alascensis shows extremely little variation in the dimensions examined even though populations occur over 12 degrees of latitude, from areas where the mean annual temperature ranges from $-12.5^{\circ} \mathrm{C}$ at Barrow to $8.8^{\circ} \mathrm{C}$ at Iliamna, the extremes range from $-60^{\circ} \mathrm{C}$ at Tanana to $+37.8^{\circ} \mathrm{C}$ at Fort Yukon, and with variations in photoperiod from the summer situation of 24 hours of sunlight in the north to 18 hours in the southwest and no hours of sunlight in mid-winter in the north to 6 hours in the southwest. 
Over this vast climatic and geographic extent, the small variation in linear dimension and the slight changes in weight are insufficient to indicate reproductive isolation of individual populations over a time period sufficient for divergence in dimensions of length.

Although we have shown that, in general, populations of $L . l$. alascensis do not differ significantly in linear dimensions throughout the range of the subspecies, there appears to be a pattern in the distribution of body weight (Table 6). The lighter individuals occur in the interior of the state along the upper and middle Yukon River Valley; weights increase in the Brooks Range towards the southwest coast and continue to increase westward to both the lower stretches of the Yukon and Kuskokwim Rivers and the northwest coast from Kotzebue to Barrow. The heaviest birds occur on Seward Peninsula. The slight differences in wing and tail lengths do not parallel the distribution of body weights and in fact show no consistent geographical trends.

Ptarmigan attributed to the subspecies $L$. l. alascensis occupy the northern and western portion of Alaska which was not covered with glacial ice at the height of Wisconsin Glaciation (Hopkins 1967). Their linear dimensions differ but little over this extensive and varied terrain. In the most northwestern and western regions the birds are heavier, showing a gradual decline toward the interior, but no significantly discrete change from one region to another (Table 6). In the central part of interior Alaska, about the Tanana Valley, and eastward in southern Yukon Territory linear dimensions and weights of ptarmigan are distinctly smaller (Table 6). The birds from Southern Yukon Territory are from the range attributed to L. l. albus, and in length and probably in weight they are like their Alaskan congeners of the central and upper Tanana Valley region. From the Tanana region into Southern Yukon Territory terrain and climate are similar. The two regions are traversed by the Alaska Highway. Probably the melting Cordilleran ice cap opened this inland route of communication eastward to the interior of Alaska early in the ablation of the Wisconsin Glaciation.

South of the range of $L . l$. alascensis in western and central Alaska and of $L . l$. albus in east central Alaska and southern Yukon Territory, L. $l$. alexandrae in Alaska occupies a region that was heavily glaciated during the Wisconsin Glaciation and perhaps then uninhabitable. These ptarmigan appear smaller than $L$. $l$. alascensis and resemble $L$. $l$. albus in dimensions. $L$. $l$. murei from Kodiak may have been derived from a stock in an unglaciated refuge on Kodiak Island. At least the birds from the island appear to be larger than $L$. l. alexandrae from the coasts of the mainland.

Our survey of dimensions of ptarmigan deals with birds collected in winter, whereas conventional taxonomy is based upon patterns of plumage in summer and more dubious estimates of size of bill. In the central Brooks Range, where we know the annual range of a homogeneous population of willow ptarmigan, migrations extend over several hundred miles and southernmost wintering is more than a hundred miles from the northern centre of nesting (Irving et al. 1967b). The mobility of component populations may account for the dimensional similarity of ptarmigan within the wide range of $L . L$. alascensis. It also points to the regularity of coherence within populations which, although capable of long migrations, 
maintains differentiation of the ptarmigan of the Tanana region from surrounding $L$. $l$. alascensis and relates the Tanana birds to $L$. $l$. albus of southern Yukon Territory.

\section{ACKNOWLEDGEMENTS}

We wish to thank the many residents of the state of Alaska for their cooperation in securing birds for our analysis. Martha Meng, Susan Savage, Ina Sledge, Joan Maggs, as well as the authors processed the many birds used in this paper. Susan Savage programmed and executed the statistical comparisons. The project was supported in part by NIH Grant GM-10402 and the State of Alaska, Department of Fish and Game, through Federal Aid in Wildlife Restoration Projects W-6-R and W-17-R, ADFG. This paper is publication No. 120 from the Institute of Arctic Biology.

\section{REFERENCES}

AMERICAN ORNIthologists UNION. 1957. Check-List of North American Birds. Baltimore, Maryland: The Lord Baltimore Press. $691 \mathrm{pp}$.

DUNCAN, D. B. 1957. Multiple range tests for correlated and heteroscedastic means. Biometrics, 13: 104-76.

Gabrielson, I. N. and F. C. LINCoLn. 1959. The Birds of Alaska. Harrisburg, Pa.: The Stackpole Co. 922 pp.

HopkINS, D. M. 1967. The Cenozoic History of Beringia - A Synthesis. In: The Bering Land Bridge, D. M. Hopkins, Editor. Stanford, California: Stanford University Press. $495 \mathrm{pp}$.

IRVING, L., G. C. WEST and L. J. PEYTON. 1967a. Winter feeding program of Alaska willow ptarmigan shown by crop contents. Condor, 69: 69-77.

IRVING, L., G. C. WEST, L. J. PEYTON and S. PANEAK. 1967b. Migration of willow ptarmigan in arctic Alaska. Arctic, 20: 77-85.

MILlER, R. G. JR. 1966. Simultaneous statistical inference. New York: McGraw-Hill Book Co. $272 \mathrm{pp}$.

WEST, G. C. 1968 . Bioenergetics of captive willow ptarmigan under natural conditions. Ecology, 49: 1035-45.

WEST, G. C. and M. S. MENG. 1968 . Seasonal changes in body weight and fat and the relation of fatty acid composition to diet in the willow ptarmigan. Wilson Bulletin, 80: 426-41.

WEST, G. C., S. SAVAGE, L. IRVING and L. J. PEYTON. 1968. Morphological homogeneity of a population of Alaska willow ptarmigan. Condor, 70: 340-47. 